\title{
Correction to: Evaluation of Generic Methods to Predict Human Pharmacokinetics Using Physiologically Based Pharmacokinetic Model for Early Drug Discovery of Tyrosine Kinase Inhibitors
}

\author{
Hong-Can Ren ${ }^{1}\left[\right.$ ? Yang Sai ${ }^{1} \cdot$ Tao Chen $^{2}$ \\ Published online: 11 September 2018 \\ (c) Springer Nature Switzerland AG 2018

\section{Correction to: \\ European Journal of Drug Metabolism and Pharmacokinetics \\ https://doi.org/10.1007/s13318-018-0496-4}

The original version of this article unfortunately contained a mistake. Conflict of interest statement was incorrect. The corrected COI statement is given below.

Dr. Chen is an employee of Shanghai PharmoGo Co., Ltd, Shanghai, an authorised distributor of Simulations Plus, the developer of GastroPlus ${ }^{\mathrm{TM}}$ and ADMET Predictor ${ }^{\mathrm{TM}}$, in China. Dr. Ren and Dr. Sai have no conflicts of interest directly relevant to the content of this study to declare.

The original article can be found online at https://doi.org/10.1007/ s13318-018-0496-4.

Hong-Can Ren

Hongcanr@hmplglobal.com

$\bowtie$ Yang Sai

Yangs@hmplglobal.com

1 Department of Clinical Pharmacology and DMPK, Hutchison MediPharma Ltd., Building 4, 720 Cailun Road, Zhang-Jiang Hi-Tech Park, Shanghai 201203, People's Republic of China

2 Shanghai PharmoGo Co., Ltd, 3F, Block B, Weitai Building, No. 58, Lane 91, Shanghai 200127,

People's Republic of China 